BULL. AUSTRAL. MATH. SOC.

$39 A 10,33 A 25$

VOL. 35 (1987) 43-48.

\title{
WEIERSTRASS ELLIPTIC DIFFERENCE EQUATIONS
}

\author{
Renfrey B. Potts
}

\begin{abstract}
The Weierstrass elliptic function satisfies a nonlinear first order and a nonlinear second order differential equation. It is shown that these differential equations can be discretized in such a way that the solutions of the resulting difference equations exactly coincide with the corresponding values of the elliptic function.
\end{abstract}

\section{Introduction.}

In a sequence of recent papers [2]-[5], it has been shown that, in choosing a difference equation $(\Delta E)$ approximation to a differential equation (DE), theoretical advantage can be obtained by exploiting a wider range of approximations than is customary.

For the simple linear second order $D E$

$$
w^{\prime \prime}(z)+w(z)=0
$$

it was shown [3] that it is 'best' to use the expression

$$
w^{\prime \prime}(z) \approx\left(w_{n+1}-2 w_{n}+w_{n-1}\right) /\left[4 \sin ^{2}(h / 2)\right]
$$

rather than the usual

$$
w^{\prime \prime}(z) \approx\left(w_{n+1}-2 w_{n}+w_{n-1}\right) / h^{2}
$$

Received 19 February 1986.

Copyright clearance Centre, Inc. Serial-fee code: 0004-9727/87 $\$ A 2.00+0.00$. 
for the approximation to the derivative.

For the nonlinear verhulst $D E$

$$
w^{\prime}(z)=w(z)-w(z)^{2}
$$

the approximation

$$
w^{\prime}(z) \approx\left(w_{n+1}-w_{n}\right) /\left(e^{h}-1\right)
$$

is the best to use [4], and with $w(z)^{2}$ replaced by $w_{n} w_{n+1}$.

For the nonlinear Duffing equation [2],

$$
w^{\prime \prime}(z)+a w(z)+b w(z)^{3}=0,
$$

the cubic term $w(z)^{3}$ is best replaced by $\frac{7}{2} b w_{n}^{2}\left(w_{n+1}+w_{n-1}\right)$.

It is the purpose of the present paper to extend this approach to the important $D E^{\prime}$ 's satisfied by the Weierstrass elliptic function $P(z)[1]$, namely the first order nonlinear $D E$ of the second degree

$$
p^{\prime}(z)^{2}=4 p(z)^{3}-g_{2} p(z)-g_{3}
$$

and the consequent second order nonlinear $D E$ of the first degree

$$
p^{\prime \prime}(z)=6 p(z)^{2}-\frac{1}{2} g_{2}
$$

The function $p(z)$ is an even function of $z, p(z)-z^{-2}$ is analytic at $z=0$ and equal to 0 at $z=0$, and the constants $g_{2}$ and $g_{3}$ are the so-called invariants.

$$
\text { Sinple } \Delta E \text { approximations to (1.7) and (1.8) are }
$$

and

$$
\left(p_{n+1}-p_{n}\right)^{2} / h^{2}=4 p_{n}^{3}-g_{2} p_{n}-g_{3}
$$

which are correct to $O(h)$. It will be shown that modifications of these can be made so that they are the 'best' $\Delta E^{\prime}$ 's possible in the sense that their solutions coincide exactly with the values of $p(z)$. More precisely, for the points

$$
\text { (1.11) } z=a+n h
$$

where $a$ is an initial value and $h$ a constant stepsize (not necessarily 'small'), then the solution $p_{n}$ of the $\Delta E^{\prime}$ 's will have the property that 


$$
p_{n}=p(a+n h)
$$

\section{First Order Difference Equation.}

The best $\triangle E$ approximation to the first order $D E$ (1.7) is obtained by using the addition formula for $p(z)$ to derive a difference equation for $p_{n}$ as defined by (1.12).

If

$$
p(h)=k
$$

then the addition formula [1] for $p(z)$ can be written

$$
p_{n+1}=\frac{1}{4}\left[\frac{p^{\prime}(z)-p^{\prime}(h)}{p_{n}-k}\right]^{2}-p_{n}-k .
$$

Solving for $p^{\prime}(z)$, namely

$$
p^{\prime}(z)=p^{\prime}(h) \pm 2\left(p_{n}-k\right)\left(p_{n+1}+p_{n}+k\right)^{\frac{1}{2}}
$$

and squaring gives

$$
-4\left(p_{n}-k\right) p_{n+1}+4 k p_{n}+8 k^{2}-g_{2}= \pm 4 p^{\prime}(h)\left(p_{n+1}+p_{n}+k\right)^{\frac{2}{2}}
$$

where use has been made of (1.7). Squaring and using (1.7) again yields the required best first order nonlinear $\Delta E$ of the second degree

$$
\begin{gathered}
\left(p_{n}-k\right)^{2}\left(p_{n+1}\right)^{2}-\left[4 k p_{n} \frac{1}{2}\left(k+p_{n}\right)-g_{2} \frac{1}{2}\left(k+p_{n}\right)-g_{3}\right] p_{n+1} \\
+\left[\left(k p_{n}+\frac{1}{4} g_{2}\right)^{2}+g_{3}\left(k+p_{n}\right)\right]=0 .
\end{gathered}
$$

To recognize this $\Delta E$ as an approximation for small $h$ to the $D E$ (1.1) requires rearranging $(2.5)$ to the form

$$
\left(p_{n+1}-p_{n}\right)^{2} k=4 p_{n} p_{n+1} \frac{1}{2}\left(p_{n}+p_{n+1}\right)-g_{2} \frac{1}{2}\left(p_{n}+p_{n+1}\right)-g_{3}
$$

which is just (2.5) with $k$ and $p_{n+1}$ interchanged.

For small $h$,

$$
k=p(h)=h^{-2}+o\left(h^{2}\right)
$$

so that

$$
\left(p_{n+1}-p_{n}\right) k^{\frac{3}{2}}=p^{\prime}(z)+o(h)
$$


and to $O(h),(2.6)$ becomes

$$
p^{\prime}(z)^{2}=4 p(z)^{3}-g_{2} p(z)-g_{3}
$$

as required.

It is interesting to note that in the difference approximation to the first derivative, the denominator used is not $h$ but $[p(h)]^{-1 / 2}$ which is $O(h)$. The replacements

$$
\begin{gathered}
p(z)^{3} \rightarrow p_{n} p_{n+1} \frac{1}{2}\left(p_{n}+p_{n+1}\right) \\
p(z) \rightarrow \frac{1}{2}\left(p_{n}+p_{n+1}\right)
\end{gathered}
$$

are not unexpected, being similar to the results previously obtained with the Duffing equation [2].

While (2.6) is valid for any $h$, the simpler result

$$
\left(p_{n+1}-p_{n}\right)^{2} / h^{2}=4 p_{n} p_{n+1} \frac{1}{2}\left(p_{n}+p_{n+1}\right)-g_{2} \frac{1}{2}\left(p_{n}+p_{n+1}\right)-g_{3}
$$

is an approximation to $O\left(h^{2}\right)$ to the $D E(1.7)$, while

$$
\left(p_{n+1}-p_{n}\right)^{2} / h^{2}=4 p_{n} p_{n+1} \frac{1}{2}\left(p_{n}+p_{n+1}\right)-g_{2} \frac{1}{2}\left(p_{n}+p_{n+1}\right)-g_{3}
$$

$$
-h^{2}\left[\left(p_{n} p_{n+1}+\frac{1}{4} g_{2}\right)^{2}+g_{3}\left(p_{n}+p_{n+1}\right)\right]
$$

is an approximation to $O\left(h^{4}\right)$.

\section{Second Order Difference Equation.}

The best $\triangle E$ approximation to the second order $D E$ (1.8) can be derived by differencing (2.6), in which, for convenience, $n$ is replaced by $n-1$. From the simple identities

$$
\begin{gathered}
\Delta\left(p_{n}-p_{n-1}\right)^{2}=\left(p_{n+1}-p_{n-1}\right)\left(p_{n+1}-2 p_{n}+p_{n-1}\right) \\
\Delta\left[p_{n-1} p_{n}\left(p_{n-1}+p_{n}\right)\right]=\left(p_{n+1}-p_{n-1}\right) p_{n}\left(p_{n+1}+p_{n}+p_{n-1}\right) \\
\Delta\left(p_{n-1}+p_{n}\right)=p_{n+1}-p_{n-1} \\
\Delta\left(p_{n-1} p_{n}+\frac{1}{4} g_{2}\right)^{2}=\left(p_{n+1}-p_{n-1}\right)\left[p_{n}^{2}\left(p_{n+1}+p_{n-1}\right)+\frac{1}{2} g_{2} p_{n}\right]
\end{gathered}
$$


Weierstrass Elliptic Difference Equations

47

follows the required best second order $\Delta E$ :

$$
\begin{aligned}
\left(p_{n+1}-2 p_{n}\right. & \left.+p_{n-1}\right) k=2 p_{n}\left(p_{n+1}+p_{n}+p_{n-1}\right)-\frac{1}{2} g_{2} \\
& -k^{-1}\left[p_{n}^{2}\left(p_{n+1}+p_{n-1}\right)+\frac{1}{2} g_{2} p_{n}+g_{3}\right] .
\end{aligned}
$$

For small $h$, this is seen to be an approximation to the $D E$ (1.8) since (2.7) gives

$$
\left(p_{n+1}-2 p_{n}+p_{n-1}\right) k=p^{\prime \prime}(z)+o(h)
$$

and

$$
2 p_{n}\left(p_{n+1}+p_{n}+p_{n-1}\right)=6 p^{\prime}(z)^{2}+o(h) .
$$

While (3.5) is valid for any $h$, the simpler $\Delta E$

$$
\left(p_{n+1}-2 p_{n}+p_{n-1}\right) / h^{2}=2 p_{n}\left(p_{n+1}+p_{n}+p_{n-1}\right)-\frac{1}{2} g_{2}
$$

is an approximation to $O\left(h^{2}\right)$ to the $D E$ (1.8), while

$$
\begin{aligned}
\left(p_{n+1}-2 p_{n}+p_{n-1}\right) / h^{2} & =2 p_{n}\left(p_{n+1}+p_{n}+p_{n-1}\right)-\frac{1}{2} g_{2} \\
& -h^{2}\left[p_{n}^{2}\left(p_{n+1}+p_{n-1}\right)+\frac{1}{2} g_{2} p_{n}+g_{3}\right]
\end{aligned}
$$

is an approximation to $O\left(h^{4}\right)$.

4. Numerical Results.

Although the main purpose of this paper is not the numerical analysis of nonlinear $D E^{\prime} s$ it is interesting to illustrate the above theory with some numerical results.

The example taken is the second order $D E$ (1.8) and its approximating $\Delta E^{\prime}$ 's $(1.10),(3.8),(3.9)$ which can be written respectively as

$$
\begin{gathered}
p_{n+1}=2 p_{n}-p_{n-1}+h^{2}\left(6 p_{n}^{2}-\frac{1}{2} g_{2}\right) \\
p_{n+1}=\left[2 p_{n}-p_{n-1}+h^{2}\left(2 p_{n}^{2}+2 p_{n} p_{n-1}-\frac{1}{2} g_{2}\right)\right] /\left(1-2 h^{2} p_{n}\right) \\
p_{n+1}=\left[2 p_{n}-p_{n-1}+h^{2}\left(2 p_{n}^{2}+2 p_{n} p_{n-1}-\frac{1}{2} g_{2}\right)\right. \\
\left.-h^{4}\left(p_{n}^{2} p_{n-1}+\frac{1}{2} g_{2} p_{n}+g_{3}\right)\right] /\left(1-h^{2} p_{n}\right)^{2} .
\end{gathered}
$$

https://doi.org/10.1017/S0004972700013022 Published online by Cambridge University Press 
With chosen values of

$$
g_{2}=8.124218, g_{3}=4.443052, h=0.05
$$

and exact values for $p(0.50)$ and $p(0.55)$, the results after 4 and 9 iterations of the $\Delta E^{\prime}$ 's gave the following:

$$
\begin{array}{ccccc}
z & \text { exact } & (4.1) & (4.2) & (4.3) \\
0.50 & 4.1124 & & & \\
0.55 & 3.4449 & & & \\
0.75 & 2.0684 & 2.051 & 2.074 & 2.0684 \\
1.00 & 1.6451 & 1.566 & 1.671 & 1.6451
\end{array}
$$

The $\Delta E^{\prime}$ 's prove to be a simple and convenient method for generating values of $p(z)$.

\section{References}

[1] A. Abramowitz and I. A. Stegun, (eds.), Hondbook of mathematical functions, U.S. National Bureau of Standards, Washington D.C. (1964).

[2] R. B. Potts, "Best difference equation approximation to Duffing's equation", J. Austral. Math. Soc. Ser. B 23 (1982), 349-356.

[3] R. B. Potts, "Differential and difference equations", Am. Math. Mon. 89 (1982), 402-407.

[4] R. B. Potts, "Nonlinear difference equation",NonIinear Anal. 6 (1982), 659-665.

[5] R. B. Potts, "Van der Pol difference equation", Noniinear Anal. 7 (1983), 801-812.

Applied Mathematics Department

The University of Adelaide

South Australia. 